April 23, 2014 13:22 WSPC/INSTRUCTION FILE "IJUFKS Bosi et al. (APRIL 2014)"

International Journal of Uncertainty, Fuzziness and Knowledge-Based Systems

(c) World Scientific Publishing Company

\title{
CONTINUOUS REPRESENTABILITY OF INTERVAL ORDERS: THE TOPOLOGICAL COMPATIBILITY SETTING
}

\author{
G. BOSI \\ Dipartimento de Scienze Economiche, Aziendali, Mathematiche e Statistiche \\ Università degli Studi di Trieste \\ Piazzale Europa 1, I-34127 \\ Trieste, Italia \\ GIANNI.BOSI@deams.units.it \\ A. ESTEVAN \\ Departamento de Matemáticas, Universidad Pública de Navarra, Campus Arrosadía \\ Pamplona, 31006, Spain \\ asier.mugertza@unavarra.es \\ J. GUTIÉRREZ GARCÍA \\ Departamento de Matemáticas, Universidad del País Vasco UPV/EHU, Apdo. 644 \\ Bilbao, 48080, Spain \\ javier.gutierrezgarcia@ehu.es

\section{E. INDURÁIN*} \\ Departamento de Matemáticas, Universidad Pública de Navarra, Campus Arrosadía \\ Pamplona, 31006, Spain \\ steiner@unavarra.es \\ Received (received date) \\ Revised (revised date)
}

In this paper, we go further on the problem of the continuous numerical representability of interval orders defined on topological spaces. A new condition of compatibility between the given topology and the indifference associated to the main trace of an interval order is introduced. Provided that this condition is fulfilled, a semiorder has a continuous interval order representation through a pair of continuous real-valued functions. Other necessary and sufficient conditions for the continuous representability of interval orders are also discussed, and, in particular, a characterization is achieved for the particular case of interval orders defined on a topological space of finite support.

Keywords: Semiorders; continuous Scott-Suppes representability

${ }^{*}$ Corresponding author. 


\section{Introduction}

Throughout the following manuscript we introduce further results on the continuous representability of interval orders, independent as well as alternative and complementary to the ones introduced in a seminal paper by Bosi et al., published in the International Journal of Uncertainty, Fuzziness and Knowledge-Based Systems in $2007 .^{1}$

The classical problem of the numerical representability of ordered structures consists in translating a given qualitative scale into a numerical or quantitative one, in order to compare numbers instead of elements of a given abstract set. ${ }^{2}$

In this approach, initiated by Cantor in 1895, the mathematical underlying problem is simple: we are given a nonempty set $X$ endowed with a binary relation (also known as qualitative scale or preference ordering) $\mathcal{R}$, and we search for realvalued functions $F: X \rightarrow \mathbb{R}$ satisfying that $x \mathcal{R} y \Leftrightarrow F(x) \leq F(y)$ for all $x, y \in X$. The aim is to reflect all the properties of $\mathcal{R}$ in terms of numbers. ${ }^{3,4,5,2}$

Nevertheless, at this point, we should notice that the mere existence of a map $F$ such that $x \mathcal{R} y \Leftrightarrow F(x) \leq F(y)$ for every $x, y \in X$, immediately forces the binary relation $\mathcal{R}$ to accomplish strong restrictive conditions, namely, in this situation $\mathcal{R}$ must be a total preorder (i.e., transitive and complete).

As a matter of fact, there are many contexts in which those conditions cannot be accomplished. For instance this happens when dealing with models in which the binary relations involved fail to be transitive. ${ }^{6,7,8,9,10}$

Therefore, the existence of a representation by means of a map $F$ with $x \mathcal{R} y \Leftrightarrow$ $F(x) \leq F(y)$ for all $x, y \in X$ must be discarded when the given binary relation fails to be a total preorder. Other different kinds of numerical representations should be used instead.

Thus, if $X$ is a nonempty set endowed with an interval order $\prec$, the classical numerical representation (provided that it exists) consists of two real-valued functions $u, v: X \rightarrow \mathbb{R}$ such that $x \prec y \Leftrightarrow v(x)<u(y)$ holds for all $x, y \in X$.

In the special case of a semiorder the classical representations, known as ScottSuppes representations in this literature consist of a real-valued map $u: X \rightarrow \mathbb{R}$ such that $x \prec y \Leftrightarrow u(x)+1<u(y)$ holds true for every $x, y \in X$. Notice that this is actually a special kind of interval order representation through a pair $(u, v)$ in which $v(t)=u(t)+1$ for every $t \in X .{ }^{11,12,13}$

Both the concepts of an interval order and a semiorder had already been introduced, at least implicitly, in the work of Wiener, but under a different nomenclature. ${ }^{14,15,16}$

Then, the notion of a semiorder is usually attributed to Luce, who reintroduced this framework of research looking for applications into Economics and Psychology. He was who coined the term in 1956. Also, the notion of an interval order is usually attributed to Fishburn, in his pioneer works on Decision Making issued in the 1970's. ${ }^{7,8,17,18,19}$

The main aim of both Luce and Fishburn was to develop mathematical models 
of measurements able to capture situations of intransitive indifference.

In addition, when the set $X$ is also endowed with a topology $\tau$, one may wonder about the semicontinuity or continuity of the numerical representations (if any) of a qualitative scale or binary relation $\mathcal{R}$ defined on $X .^{20,21,22,23}$

The existence of a pair of upper semicontinuous real-valued functions representing an interval order on a topological space has recently been characterized by Bosi and Zuanon. In that direction, such a characterization, which generalizes previous interesting results presented by Bridges, is the most general one, since the authors do not impose any conditions neither on the topology nor on the two functions that make up the representation. ${ }^{24,25}$

The problem of finding a characterization of the continuous representability of an interval order $\prec$ defined on a topological space $(X, \tau)$ remains still open.

However, important results have ben obtained whenever the topology $\tau$ is natural as regards the interval order (see the Section 3 below) through an ordinal condition called interval order separability (see Section 2). ${ }^{1}$

The continuity here is understood with respect to the topology $\tau$ on $X$ and the usual (Euclidean) topology $\tau_{u}$ on the real line $\mathbb{R}$.

Other (partial) results about continuous representability of interval orders may be seen in this literature. ${ }^{26,27,28,29,30,31}$

The problem of characterizing the continuous Scott-Suppes representability of a semiorder also remains open. Furthermore, unlike interval orders, in this case of semiorders the concept of a natural topology does not furnish good results, in general. ${ }^{1,32}$

Some (partial) results about continuous representability of semiorders have also been introduced ${ }^{33,34,32}$

But even with this negative output, there is an important and hopefully, quite positive fact: the analysis of the properties related to semiorders defined on a topological space have lead to a key concept, namely that of the topological compatibility with respect to the indifference of the main trace. Moreover, this new concept can be introduced not only for semiorders, but, actually, for the more general case of interval orders, that constitutes the nucleus of our studies throughout the present manuscript. As we analyze in Section 3, by means of this notion we obtain new results on continuous representability of interval orders, valid for topologies that may even fail to be natural.

The structure of the paper goes as follows:

After the Introduction and Preliminaries, in Section 3 we introduce new results on continuous representability of interval orders, that are valid for topologies compatible with the indifference of the main trace of the given interval ordered structure. In Section 4 we analyze the continuous representability of interval orders defined on topological spaces $(X, \tau)$ in which the set $X$ is finite. A section of final remarks closes the paper. 


\section{Preliminaries}

\subsection{Basic definitions and notation}

From now on $X$ will denote a nonempty set.

Definition 1. A binary relation $\mathcal{R}$ on $X$ is a subset of the Cartesian product $X \times X$. Given two elements $x, y \in X$, we will use the standard notation $x \mathcal{R} y$ to express that the pair $(x, y)$ belongs to $\mathcal{R}$.

Associated to a binary relation $\mathcal{R}$ on a set $X$, we consider its negation (respectively, its transpose) as the binary relation $\mathcal{R}^{c}$ (respectively, $\mathcal{R}^{t}$ ) on $X$ given by $(x, y) \in \mathcal{R}^{c} \Leftrightarrow(x, y) \notin \mathcal{R}$ for every $x, y \in X$ (respectively, given by $(x, y) \in \mathcal{R}^{t} \Leftrightarrow$ $(y, x) \in \mathcal{R}$, for every $x, y \in X)$. We also define the adjoint $\mathcal{R}^{a}$ of the given relation $\mathcal{R}$, as $\mathcal{R}^{a}=\left(\mathcal{R}^{t}\right)^{c}$.

A binary relation $\mathcal{R}$ defined on a set $X$ is said to be:

(i) reflexive if $x \mathcal{R} x$ holds for every $x \in X$,

(ii) irreflexive if $\neg(x \mathcal{R} x)$ holds for every $x \in X$,

(iii) symmetric if $\mathcal{R}$ and $\mathcal{R}^{t}$ coincide,

(iv) antisymmetric if $\mathcal{R} \cap \mathcal{R}^{t} \subseteq \Delta=\{(x, x): x \in X\}$,

(v) asymmetric if $\mathcal{R} \cap \mathcal{R}^{t}=\varnothing$,

(vi) total if $\mathcal{R} \cup \mathcal{R}^{t}=X \times X$,

(vii) transitive if $x \mathcal{R} y \wedge y \mathcal{R} z \Rightarrow x \mathcal{R} z$ for every $x, y, z \in X$.

In the particular case of a nonempty set where some kind of ordering has been defined, the standard notation is different. We include it here for the sake of completeness, since we will use it throughout the present manuscript.

Definition 2. A preorder $\precsim$ on $X$ is a binary relation on $X$ which is reflexive and transitive. An antisymmetric preorder is said to be an order. A total preorder $\precsim$ on a set $X$ is a preorder such that if $x, y \in X$ then $(x \precsim y) \vee(y \precsim x)$ holds. If $\precsim$ is a preorder on $X$, then as usual we denote the associated asymmetric relation by $\prec$ and the associated equivalence relation by $\sim$ and these are defined by $x \prec y \Leftrightarrow$ $(x \precsim y) \wedge \neg(y \precsim x)$ and $x \sim y \Leftrightarrow(x \precsim y) \wedge(y \precsim x)$.

Definition 3. An interval order $\prec$ is an asymmetric binary relation on $X$ such that $(x \prec y) \wedge(z \prec t) \Rightarrow(x \prec t) \vee(z \prec y)(x, y, z, t \in X)$. Its corresponding adjoint will be denoted $\precsim$, so that $a \precsim b \Leftrightarrow \neg(b \prec a)$. This relation $\precsim$ is called the weak preference associated to $\prec$. By the way, $\prec$ is also called a strict preference defined on $X$. In addition, the binary relation $\sim$ defined by $a \sim b \Leftrightarrow(a \precsim b) \wedge(b \precsim a)$ is said to be the indifference associated to $\prec$.

Remark 1. It is well known that given an interval order $\prec$ on a set $X$, the associated relations $\precsim$ and $\sim$ may fail to be transitive. ${ }^{11,12,7,8,19}$

Definition 4. An interval order $\prec$ is said to be a semiorder if $(x \prec y) \wedge(y \prec$ $z) \Rightarrow(x \prec w) \vee(w \prec z)(x, y, z, w \in X)$. A semiorder $\prec$ is said to be typical if its 
associated weak preference $\precsim$ defined by $x \precsim y \Leftrightarrow \neg(y \prec x),(x, y \in X)$ is not a total preorder on $X$.

\subsection{The traces and the numerical representability of interval orders and semiorders}

Through the next definition, we introduce the notion of representability for different kinds of orderings. The underlying idea corresponds to the possibility of converting qualitative scales into quantitative ones.

Definition 5. A total preorder $\precsim$ on $X$ is called representable if there is a realvalued function $u: X \rightarrow \mathbb{R}$ that is order-preserving, so that, for every $x, y \in X$, it holds that $x \precsim y \Leftrightarrow u(x) \leq u(y)$. The map $u$ is said to be a utility function for $\precsim$.

An interval order $\prec$ defined on $X$ is said to be representable (as an interval order) if there exist two real valued maps $u, v: X \longrightarrow \mathbb{R}$ such that $x \prec y \Leftrightarrow v(x)<$ $u(y) \quad(x, y \in X)$. The pair $(u, v)$ is called a utility pair representing $\prec$.

A semiorder $\prec$ defined on $X$ is said to be representable in the sense of Scott and Suppes if there exists a real-valued map $u: X \rightarrow \mathbb{R}$ (again called a utility function) such that $x \prec y \Leftrightarrow u(x)+1<u(y)(x, y \in X){ }^{12}$

In this case, the pair $(u, 1)$ is said to be a Scott-Suppes representation of $\prec$.

Remark 2. If $(u, v)$ is utility pair representing an interval order $\prec$ defined on a set $X$, it is straightforward to see that $u(x) \leq v(x)$ for every $x \in X$. The real interval $[u(x), v(x)]$, that could degenerate to a point if $u(x)=v(x)$, is said to be the interval of discrimination or perception corresponding to the element $x \in X$. And the non-negative real number $v(x)-u(x)$ is said to be the discrimination threshold for the element $x \in X$. Notice that these thresholds depend on the elements of $X$. If $x \neq y \in X$ it may happen that $v(x)-u(x) \neq v(y)-u(y)$. In the case of a semiorder that is representable in the sense of Scott and Suppes, the discrimination thresholds are all equal to 1 .

There exist interval orders that fail to be representable (as interval orders). Also, there exist semiorders that are not representable in the sense of Scott and Suppes. ${ }^{35,36}$

Definition 6. Associated to an interval order $\prec$ defined on a nonempty set $X$, we shall consider three new binary relations. ${ }^{17,7,37}$

These binary relations are said to be the traces of $\prec$. They are respectively denoted by $\prec^{*}$ (left trace), $\prec^{* *}$ (right trace) and $\prec^{0}$ (main trace), and defined as follows: $x \prec^{*} y \Leftrightarrow x \prec z \precsim y$ for some $z \in X$, and similarly $x \prec^{* *} y \Leftrightarrow x \precsim z \prec y$ for some $z \in X \quad(x, y \in X)$. In addition, $x \prec^{0} y \Leftrightarrow\left(x \prec^{*} y\right) \vee\left(x \prec^{* *} y\right) \quad(x, y \in X)$.

Remark 3. We denote $x \precsim^{*} y \Leftrightarrow \neg\left(y \prec^{*} x\right), \quad x \sim^{*} y \Leftrightarrow x \precsim^{*} y \precsim^{*} x, \quad x \precsim^{* *} y \Leftrightarrow$ $\neg\left(y \prec^{* *} x\right)$ and $x \sim^{* *} y \Leftrightarrow x \precsim^{* *} y \precsim^{* *} x$, and finally $x \precsim^{0} y \Leftrightarrow\left(x \precsim^{*} y\right) \wedge\left(x \precsim^{* *}\right.$ $y)$ and $x \sim^{0} y \Leftrightarrow\left(x \precsim^{0} y\right) \wedge\left(y \precsim^{0} x\right) \quad(x, y \in X)$. Both the binary relations $\precsim^{*}$ and $\precsim$ ${ }^{* *}$ are total preorders on $X$. Moreover, the indifference relation $\sim$ associated to 
the interval order $\prec$ is transitive if and only if $\precsim^{*}, \precsim^{* *}$ and $\precsim$ coincide. In this case $\precsim$ is actually a total preorder on $X{ }^{17,7,38,35,39}$

In addition, the binary relation $\precsim^{0}$ allows us to characterize semiorders among interval orders. ${ }^{17,7,38}$

Indeed, if $\prec$ an interval order on $X$, then it is a semiorder if and only if $\precsim^{0}$ is a total preorder on $X$. A semiorder $\prec$ is not typical if and only if $\precsim^{*}, \precsim^{* *}, \precsim^{0}$ and $\precsim$ coincide.

Let us recall now some characterizations of the numerical representability of total preorders, interval orders and semiorders.

Definition 7. Let $X$ be a nonempty set. A total preorder $\precsim$ defined on $X$ is said to be perfectly separable if there exists a countable subset $D \subseteq X$ such that for every $x, y \in X$ with $x \prec y$ there exists $d \in D$ such that $x \precsim d \precsim y$.

An interval order $\prec$ defined on $X$ is said to be interval order separable if there exists a countable subset $D \subseteq X$ such that for every $x, y \in X$ with $x \prec y$ there exists $d \in D$ such that $x \precsim^{*} d \prec y$.

A semiorder $\prec$ defined on $X$ is said to be regular with respect to sequences if for any $x, y \in X$, and sequences $\left(x_{n}\right)_{n \in \mathbb{N}},\left(y_{n}\right)_{n \in \mathbb{N}} \subseteq X$, none of the situations $x \prec \ldots \prec x_{n+1} \prec x_{n} \prec \ldots \prec x_{1}$ and $y_{1} \prec \ldots \prec y_{n} \prec y_{n+1} \prec \ldots \prec y$ may occur.

The following facts are well-known in this literature. ${ }^{2,35,39}$

Theorem 1. On a nonempty set $X$ the following statements hold true:

(a) A total preorder $\precsim$ is representable if and only if it is perfectly separable.

(b) An interval order $\prec$ is representable if and only if it is interval order separable.

And the following result has recently been proved..$^{40,13}$

Theorem 2. Let $X$ be a nonempty set. Let $\prec$ be a typical semiorder defined on $X$. Then, $\prec$ is representable in the sense of Scott and Suppes if and only if it is both interval order separable and regular with respect to sequences.

Remark 4. When $X$ is countable, the condition of interval order separability trivially holds. Therefore, a semiorder $\prec$ on a countable set $X$ is representable in the sense of Scott and Suppes if and only if it is regular with respect to sequences. This fact was already known. ${ }^{41,42}$

\section{New trends on the continuous representability of interval orders}

Let $(X, \tau)$ stand for a topological space (a set $X$ with a topology $\tau$ ).

\subsection{Background on continuous representability}

Definition 8. Let $\prec$ denote an asymmetric binary relation on $X$. Given $a \in X$ the sets $L(a)=\{t \in X: t \prec a\}$ and $U(a)=\{t \in X: a \prec t\}$ are called, respectively, 
the lower and upper contours of $a$ relative to $\prec$. We say that $\prec$ is $\tau$-continuous if for each $a \in X$ the sets $L(a)$ and $U(a)$ are $\tau$-open.

Definition 9. Let $\prec$ be an interval order on $X$. Then the topology $\tau$ is said to be natural for $\prec$ if its associated asymmetric binary relation $\prec$, as well as both the traces $\prec^{*}$ and $\prec^{* *}$, are all $\tau$-continuous. ${ }^{1}$

Remark 5. In particular, if $\precsim$ is a total preorder on $X$, so that $\precsim, \precsim^{*}$ and $\precsim^{* *}$ coincide, the topology $\tau$ is said to be natural for $\precsim$ if its associated asymmetric binary relation $\prec$ is $\tau$-continuous. (in this case, we also say that the total preorder $\precsim$ is $\tau$-continuous). For this particular case of total preorders, the notion of a natural topology was already introduced by Debreu. ${ }^{43,44}$

The following results on continuous representability are well-known in this literature. ${ }^{43,44,2,1}$

\section{Theorem 3.}

(a) A total preorder $\precsim$ on a topological space is representable through a continuous utility function if and only if it $\precsim$ is perfectly separable and the topology $\tau$ is natural for .

(b) Let $(X, \tau)$ be a topological space endowed with an interval order $\prec$ such that the topology $\tau$ is natural for $\prec$. Then $\prec$ is representable by means of a pair of continuous real-valued functions $(u, v)$ such that, in addition, $u$ (respectively, $v)$ is a utility function for the total preorder ${ }^{* *}$ (respectively, ${ }^{*}$ ), if and only if $\prec$ is interval order separable.

From Theorem 3 and Theorem 1, next Corollary 1 is easily achieved.

Corollary 1. Let $(X, \tau)$ be a topological space. Suppose that $X$ is endowed with a representable total preorder $\prec$ (respectively, with a representable interval order $\prec)$. Assume also that the topology $\tau$ is natural for $\precsim$ (respectively, for $\prec)$. Then $\precsim$ (respectively, $\prec$ ) is continuously representable.

\section{Example 1.}

(a) It may still happen that an interval order $\prec$ on a topological space $(X, \tau)$ has a representation through a pair of continuous real-valued functions, but the topology $\tau$ fails to be natural with respect to $\prec$. Thus, let $X=[2,3] \cup[9,10]$. Let $\tau$ denote the restriction to $X$ of the usual Euclidean topology of the real line $\mathbb{R}$. Endow $X$ with the interval order $\prec$ defined by $x \prec y \Leftrightarrow x^{2}<y$, for all $x, y \in X$. It is plain that the pair $u, v: X \rightarrow \mathbb{R}$ where $u(x)=x$ and $v(x)=$ $x^{2}(x \in X)$ is a representation of the interval order $\prec$. Moreover, both $u$ and $v$ are continuous functions. But $\tau$ is not natural for $\prec$. To see this, notice that 
$\left\{z \in X: z \prec^{* *} 9.5\right\}=\{9\} \cup[2,3]$, as well as $\left\{y \in X: 2.5 \prec^{*} y\right\}=\{3\} \cup[9,10]$ are not $\tau$-open. (See Ref. 1 and Remark 11 in Ref. 32).

(b) Let $(X, \tau)$ be a topological space endowed with a semiorder $\prec$. Suppose that, considering $\prec$ as an interval order, the topology $\tau$ is natural for $\prec$. Even in this situation, we cannot adapt part (b) of Theorem 3 to the semiorder case, in general. To see this, let $(u, 1)$ be a continuous Scott-Suppes representation of the semiorder $\prec$. Let $v: X \rightarrow \mathbb{R}$ be the function defined as $v(x)=u(x)+1(x \in X)$. Observe that if we want $u$ to represent $\precsim^{* *}$ and $v$ to represent $\precsim^{*}$, then $\precsim^{*}$ and $\precsim^{* *}$ must coincide, a fortiori. But, in general, $\prec^{*}$ and $\prec^{* *}$ do not agree: consider, for instance, $X=[0,3] \subset \mathbb{R}$ endowed with the semiorder $\prec$ given by $x \prec y \Leftrightarrow x+1<y(x, y \in X)$. Notice that $2 \precsim 1 \prec 3$ so that $2 \prec^{* *} 3$, but $2 \prec^{*} 3$ does not hold because there is no $z \in X$ such that $2 \prec z$. (See Remark 12 in Ref. 32).

(c) It may also happen that an interval order $\prec$ is representable through a pair of continuous functions $(u, v)$ but $u$ does not represent $\precsim^{* *}$ or $v$ does not represent $\precsim^{*}$. Actually, a glance at part (b) of this Example 1 shows that the pair $(u, v)$ with $u(x)=x$ and $v(x)=x+1$ for every $x \in X$ represents $\prec$ but $v$ does not represent $\precsim *$ since $v(2)=3<v(3)=4$ while $2 \prec^{*} 3$ does not hold.

\subsection{Topological compatibility with respect to the indifference of the main trace of an interval order}

The following facts are straightworward consequences of the definition of the traces of an interval order $\prec$ on a set $X$.

Lemma 1. Let $\prec$ be an interval order on a set $X$. The following statements hold true for every $x, y \in X$ :

(a) $x \precsim^{*} y$ holds if and only if for all $z \in X$ we have that $y \prec z \Rightarrow x \prec z$. In terms of upper contours: $x \precsim * y \Leftrightarrow U(y) \subseteq U(x)$.

(b) $x \precsim \precsim^{* *} y$ holds if and only if for all $z \in X$ we have that $z \prec x \Rightarrow z \prec y$. In terms of lower contours: $x \precsim * * y \Leftrightarrow L(x) \subseteq L(y)$.

Lemma 1 gives rise to the following key result.

Theorem 4. Let $\prec$ be an interval order defined on a set $X$. Then the indifference $\sim^{0}$ associated to the main trace is an equivalence relation.

Proof. Just notice that, by Lemma 1 we have $x \sim^{0} y \Leftrightarrow(U(x)=U(y)) \wedge(L(x)=$ $L(y))$, for all $x, y \in X$.

Remark 6. Observe that this equivalence is not only valid when $\prec$ is a semiorder, but actually in the more general case of an interval order. That is, given an interval order $\prec$, it may happen that $\precsim^{0}$ is not transitive, so that $\prec$ fails to be a semiorder. Nevertheless, $\sim^{0}$ is always transitive, because it is an equivalence. 
This Remark 6 induces the following key definition, on which the main results in this Section 3 lean.

Definition 10. Let $(X, \tau)$ be a topological space. Let $\prec$ be an interval order on $X$. The topology $\tau$ is said to be compatible with respect to the indifference of the main trace of $\prec$ if $x \sim^{0} y \Rightarrow(x \in \mathcal{O} \Longleftrightarrow y \in \mathcal{O})$ holds true for every $x, y \in X$ and every $\tau$-open subset $\mathcal{O} \in \tau$.

Remark 7. The main fact involved in this last definition is that elements that are indistinguishable with respect to $\prec$ (because all their corresponding contours agree) should also be indistinguishable from a topological point of view since, with respect to the topology $\tau$, they should (a fortiori) belong to the same $\tau$-open subsets.

Theorem 3 does not give information about continuous representability of semiorders in the sense of Scott and Suppes. In this direction, we introduce now some (partial) result, stated in terms of the topological compatibility just defined.

To start with, we recall some necessary conditions for the continuous ScottSuppes representability of a semiorder, already stated in Ref. 32 .

Lemma 2. Let $(X, \tau)$ be a topological space endowed with a semiorder $\prec$. Assume that $\prec$ is representable in the sense of Scott and Suppes by means of a pair $(u, 1)$ with $u$ continuous. Then the following properties hold true:

(a) The semiorder $\prec$ is $\tau$-continuous.

(b) If a net $\left(x_{j}\right)_{j \in J} \subseteq X$ converges to two points $a, b \in X$, then $a \sim^{0} b$.

(c) If a net $\left(x_{j}\right)_{j \in J} \subseteq X$ converges to $a \in X$, and $b, c \in X$ are such that $x_{j} \prec b \precsim a$ and also $x_{j} \prec c \precsim a$ for every $j \in J$, then $b \sim^{0} c$.

(d) If a net $\left(x_{j}\right)_{j \in J} \subseteq X$ converges to $a \in X$, and $b, c \in X$ are such that $a \precsim b \prec x_{j}$ and also $a \precsim c \prec x_{j}$ for every $j \in J$, then $b \sim^{0} c$.

Definition 11. Let $(X, \tau)$ be a topological space endowed with a semiorder $\prec$. We say that the semiorder $\prec$ is suitable (as regards $\tau$ ) if it satisfies all the necessary conditions introduced in Lemma 2.

And, as announced before, a new necessary condition appears now, and it is stated in terms of topological compatibility with respect to the main trace of a semiorder.

Proposition 1. Let $(X, \tau)$ be a topological space endowed with a semiorder $\prec$. Assume that $\tau$ is compatible with respect to the indifference of the main trace of $\prec$. Suppose also that $\prec$ is representable in the sense of Scott and Suppes by means of a pair $(u, 1)$ with $u$ continuous. Then the total preorder $\precsim^{0}$ is $\tau$-continuous.

Proof. For any $x, y \in X$ such that $x \prec^{0} y$, there is some $z \in X$ such that $x \prec z \precsim y$ or $x \precsim z \prec y$ holds. Hence $u(x)<u(z)+1 \leq u(y)$ or $u(x) \leq u(z)+1<u(y)$ holds, so we get $u(x)<u(y)$ in any case. 
Assume now that $x \precsim^{0} y$. In this situation, two cases my occur: If $y \prec^{0} x$ we already know that $u(y)<u(x)$. If $x \sim^{0} y$, by the compatibility of $\tau$ with respect to $\sim^{0}, x$ and $y$ belong to the same $\tau$-open subsets. In particular, any constant net $(x)_{\alpha \in A}$ converges both to $x$ and $y$. Thus, by continuity of $u$, we have that the constant net $(u(x))_{\alpha \in A}$ converges in the usual Euclidean topology of the real line $\mathbb{R}$ both to $u(x)$ and $u(y)$. Hence $u(x)=u(y)$, because the usual topology of $\mathbb{R}$ is Hausdorff, so that limits are unique. (Here $A$ stands for a directed set of indices).

Thus $u$ is actually a continuous representation for the total preorder $\precsim^{0}$. Therefore, by part (a) of Theorem 3, the topology $\tau$ is natural for $\precsim^{0}$. In other words: the total preorder $\precsim^{0}$ is $\tau$-continuous.

\section{Remark 8.}

(1) Let $\prec$ be a semiorder defined on a topological space $(X, \tau)$. Assume thus, viewing $\prec$ as an interval order, the topology $\tau$ is natural with respect to $\prec$. Then the total preorder $\precsim^{0}$ is $\tau$-continuous, by definition of the main trace $\prec^{0}$. The converse implication is not true in general (see Example 4 later on).

(2) In general, for any semiorder defined on a topological space $(X, \tau)$, the $\tau$ continuity of the total preorder $\precsim^{0}$ is not a necessary conditon for the continuity of the representation (see Example 2).

Example 2. Let $X=[0,1) \bigcup\left\{1^{\prime} 5\right\}$ endowed with the usual topology inherited from the Euclidean topology on $\mathbb{R}$. Let $\prec$ be the semiorder on $X$ defined by $x \prec y \Leftrightarrow x+$ $1<y$. Notice that the quotient space $X / \sim^{0}$ is finite. Indeed $X / \sim^{0}=\left\{\overline{0}, \overline{0^{\prime}} \overline{1^{\prime} 5}\right\}$, with $\overline{0}=\left[0,0^{\prime} 5\right), \overline{0^{\prime} 5}=\left[0^{\prime} 5,1\right), \overline{1^{\prime} 5}=\left\{1^{\prime} 5\right\}$. The quotient topology fails to be discrete, since $\overline{0^{\prime} 5}$ is not open. Therefore $\precsim^{0}$ cannot be $\tau$-continuous: the reason is that in case of $\tau$-continuity it would induce a continuous linear order on the quotient $X / \sim^{0}$, that is finite. But, at this point, is is well-known the only topology on a finite set for which a linear order is continuous is the discrete one. As a matter of fact, in this example we have that any Scott-Suppes representation $(u, 1)$ of the semiorder $\prec$ such that, in addition, $u$ represents the total preorder $\precsim^{0}$, would fail to be continuous: the reason is that the preimage of a small enough neighborhood of $0^{\prime} 5$ is $\left[0^{\prime} 5,1\right)$, which is not $\tau$-open. However, we may notice that $\prec$ is actually continuous representable in the sense of Scott and Suppes, through the pair $(i, 1)$ where $i: X \rightarrow \mathbb{R}$ is the inclusion map $(i(x)=x$ for every $x \in X)$.

Looking for a sort of converse of Proposition 1, we will consider a semiorder that is already representable in the sense of Scott and Suppes. We wonder when that semiorder has a continuous representation. In this direction, we have got a partial result (namely Theorem 5 below), that guarantees the continuous representability of a semiorder but as an interval order (that is, through a pair $(u, v)$ of continuous real-valued functions) provided that the condition of compatibility between the topology $\tau$ and the indifference $\sim^{0}$ is met. 
To prove the announced result, first we need to introduce a preparatory lemma.

Lemma 3. Let $X$ be a nonempty set. Let $\prec$ be a semiorder on $X$. Assume that $\prec$ is representable in the sense of Scott and Suppes. Then there exists a pair $(u, 1)$ that represents $\prec$ and, in addition, the utility function $u$ represents the total preorder $\precsim 0$

Proof. See Lemma 3.2 and Remark 3.3 in Ref. 13.

By the way, Lemma 3 can not be extended to the continuous case, as next Example 3 shows.

Example 3. Considering again the semiorder $\prec$ introduced in Example 1 (a), we may observe that the equivalence classes in $X / \sim^{0}$ are $\overline{2}=[2,3) ; \overline{3}=\{3\} ; \overline{9}=\{9\}$ and $\overline{10}=(9,10]$. Moreover $x \prec y$ for every $x \in \overline{2}$ and $y \in \overline{9} \cup \overline{10} ; x \sim y$ for every $x \in \overline{2}$ and $y \in \overline{3} ; x \prec y$ for every $x \in \overline{3}$ and $y \in \overline{10} ; x \sim y$ for every $x \in \overline{3}$ and $y \in \overline{9}$ and, finally, $x \sim y$ for every $x \in \overline{9}$ and $y \in \overline{10}$. Hence $\prec$ is actually a semiorder on $X$. But this semiorder does not admit a continuous Scott-Suppes representation that in addition, also represents the main trace $\precsim^{0}$ : again, the reason is that the quotient topology in $X / \sim^{0}$ is not the discrete one. However, it actually admits a continuous Scott-Suppes representation, namely the pair $(u, 1)$ with $u(x)=x$ for all $x \in[2,3]$ and $u(x)=x-5$ for every $x \in[9,10]$.

Remark 9. Notice that in Lemma 3 we are not saying that if the pair $(u, 1)$ constitutes a Scott-Suppes representation of $\prec$ then $u$ represents $\precsim^{0}$. What Lemma 3 states is that we can find another Scott-Suppes representation $\left(u^{\prime}, 1\right)$ where $u^{\prime}$ could perhaps be different from $u$, such that now $u^{\prime}$ is a utility function for the total preorder $\precsim 0$.

However, in the particular case in which the pair $(u, 1)$ is a Scott-Suppes representation of $\prec$, with $u$ continuous and the topology $\tau$ being compatible as regards $\sim^{0}$, then it is indeed true that the given function $u$ also represents $\precsim^{0}$. This fact has been proved in Proposition 1.

Theorem 5. Let $(X, \tau)$ be a topological space. Let $\prec$ be a representable semiorder on $X$. Assume that $\prec$ is suitable as regards $\tau$ and, in addition, the topology $\tau$ is compatible with respect to the indifference of the main trace of $\prec$. If $\precsim 0$ is $\tau$ continuous, then $\prec$ admits a representation as an interval order, through a pair $(u, v)$ of continuous real-valued functions.

Proof. Taking into account the result stated in Lemma 3, first we consider a realvalued function $f: X \rightarrow \mathbb{R}$ such that the pair $(f, 1)$ is a Scott-Suppes representation of $\prec$, with the additional condition of the map $f$ being a utility function for the total preorder $\precsim^{0}$. Define $g: X \rightarrow \mathbb{R}$ as $g(x)=f(x)+1 \quad(x \in X)$. Obviously, $g$ is also a utility function for $\precsim^{0}$. These functions $f$ and $g$ define on $\mathbb{R}$ the subsets $f(X)$ and $g(X)$. Since $\precsim^{0}$ is $\tau$-continuous, $f$ is continuous at every point of $X$, with the 
only possible exception of some points $x \in X$ such that $(f(x), b]$ or $[b, f(x))$ is a gap of $f(X)$ for some $b \in \mathbb{R}$. (See e.g. the discussion on pp. 38 and 39, Section 3.1 in Ref. 2, for further details). The analogous fact is also valid for $g$, mutatis mutandis. Let $D_{f}$ (respectively, $D_{g}$ ) denote the subset of $X$ in which $f$ (respectively, $g$ ) fails to be continuous. A gap of $f(X)$ of the type $(a, b]$ or $[b, a)(a, b \in \mathbb{R})$ is said to be badif there exists some $x \in X$ such that $f(x)=a$ and $f$ is discontinuous at $x$.

Let $B_{f}=\{t \in \bigcup I: I$ is a bad gap of $f(X)\}$. Endow $\mathbb{R} \backslash B_{f}$ with the order topology $\tau_{\leq}$corresponding to the usual ordering $\leq$on $\mathbb{R} \backslash B_{f}$, as a subset of the real line. The inclusion function that goes from $\left(\mathbb{R} \backslash B_{f}, \tau_{\leq}\right)$to $\left(\mathbb{R}, \tau_{u}\right)$ is then continuous at every point, excluding those in $f\left(D_{f}\right)$. Notice that $\mathbb{R} \backslash B_{f}$ has neither a left bound nor a right bound. In addition, $f(X) \subseteq \mathbb{R} \backslash B_{f}$ and both subsets $f(X)$ and $\mathbb{R} \backslash B_{f}$ share the bad gaps. Moreover the final topology $\tau_{f i n(f)}$ on $f(X)$ corresponding to the function $f:(X, \tau) \rightarrow f(X)$ is finer than the restriction to $f(X)$ of the order topology $\tau_{\leq}$of $\mathbb{R} \backslash B_{f}$. Accordingly, any continuous map defined on $\left(\mathbb{R} \backslash B_{f}, \tau_{\leq}\right)$ will also be be continuous when considered as a map defined on $\left(f(X), \tau_{f i n(f)}\right)$.

By the so-called Debreu' s Gap Lemma (see e.g., Lemma 3.1.4 on pp. 42-43 of the key Ref. 2 or, alternatively, see Theorem 1 in Ref. 45), there exists a strictly increasing real valued function $h: \mathbb{R} \backslash B_{f} \rightarrow \mathbb{R}$ such that the gaps of $h\left(\mathbb{R} \backslash B_{f}\right)$ are all open and, in addition, the function $h:\left(f(X), \tau_{f i n(f)}\right) \rightarrow\left(\mathbb{R}, \tau_{u}\right)$ is continuous. Hence the composition $h \circ f:(X, \tau) \rightarrow\left(\mathbb{R}, \tau_{u}\right)$ is also continuous.

Let us see now that we can extend $h$ to the bigger domain $g(X) \cup\left(\mathbb{R} \backslash B_{f}\right)$ in a way that the extended function $\bar{h}$ satisfies that the composition $\bar{h} \circ f=h \circ f$ : $(X, \tau) \rightarrow\left(\mathbb{R}, \tau_{u}\right)$ is continuous ${ }^{1}$, and the pair $(\bar{h} \circ f, \bar{h} \circ g)$ is actually an interval order representation of $\prec$. To prove this, fix a point $t \in g(X)$. The following situations may occur:

Case 1: If $t \in g(X) \bigcap\left(\mathbb{R} \backslash B_{f}\right)$, we declare that $\bar{h}(t)=h(t)$. In particular, $\bar{h}$ will become an extension of $h$.

Case 2: If $t \in g(X) \bigcap B_{f}$ belongs to a bad gap of $f(X)$ of the kind $[b, a)$ for some real numbers $a, b$, with $f(x)=a$ for some $x \in X$ such that $f$ is discontinuous at $x$, then $g(y)=t<a=f(x)$ holds for some $y \in X$. Thus $y \prec x$. Since the semiorder $\prec$ is, by hypothesis, suitable, it is in particular $\tau$-continuous (see part (a) of Lemma 2). Thus, the set $U_{\prec}(y)=\{z \in X: g(y)<f(z)\}=$ $f^{-1}([a,+\infty))=f^{-1}([f(x),+\infty))$ is $\tau$-open. Moreover, for any $c \in \mathbb{R}$ such that $a=f(x)<c$ it holds that $f^{-1}([a, c))=f^{-1}([f(x),+\infty)) \bigcap f^{-1}([-\infty, c))$. As a matter of fact, $f^{-1}([-\infty, c))=\bigcup\left\{L_{\prec 0}(z): f(z)<c\right\}$ since $f$ is a utility function that represents $\precsim^{0}$. Thus $f^{-1}([-\infty, c)$ is $\tau$-open because, by hypothesis, the main trace $\precsim^{0}$ had been assumed to be $\tau$-continuous. Therefore $f^{-1}([a, c))$ is $\tau$-open for every $c \in \mathbb{R}$ such that $a=f(x)<c$. This implies that $f$ is continuous

$\overline{{ }^{1} \text { Notice that in general } \bar{h} \neq h \text {, but } \bar{h} \circ f}=h \circ f$. In addition, $\bar{h} \circ f=h \circ f$ is continuous by the Debreu's gap Lemma. 
at $x$, which contradicts the assumption of $[b, a)$ being a bad gap of $f(X)$. In other words, case 2 is impossible.

Case 3: If $t \in g(X) \cap B_{f}$ belongs to a bad gap of $f(X)$ of the kind $(a, b]$ for some real numbers $a, b$, with $f(x)=a$ for some $x \in X$ such that $f$ is discontinuous at $x$, then it is straightforward to see that there exists a net $\left(x_{\alpha}\right)_{\alpha \in A^{2}}$ in $X$ such that $a<b<f\left(x_{\alpha}\right)$ holds for every $\alpha \in A$, and $\left(x_{\alpha}\right)_{\alpha \in A}$ converges to $x$. Since $\prec$ is a suitable semiorder, it follows by part (d) of Lemma 2 that $\{t\}=g(X) \bigcap[a, b]$. In this situation, we define $\bar{h}(t)=h(a)$.

Once the extension $\bar{h}$ has been defined, we check that $g(x)<f(y) \Leftrightarrow \bar{h}(g(x))<$ $\bar{h}(f(y))=h((f(y))$ holds true for every $x, y \in X$. To see this, we may observe that if $g(x) \in f(X)$ then the claim is obvious because $h$ is strictly increasing in $f(X)$. Furthermore, if $g(x) \notin f(X)$ then $g(x)$ belongs to a gap $(f(z)=a, b]$ for some $z \in X$ and $a, b \in \mathbb{R}$. Therefore, for any $y \in X$ with $f(y)>g(x)$ it holds that $f(y)>f(z)=a$, and then $h(f(y))>h(a)=\bar{h}(g(x))$.

Notice also that $\bar{h}$ is strictly increasing, not only in $\mathbb{R} \backslash B_{f}$ but also in $g(X)$, so that each of the functions $\bar{h} \circ f(=h \circ f)$ and $\bar{h} \circ g$ is a utility representation for the total preorder $\precsim^{0}$ associated to the main trace of the given semiorder $\prec$.

At this stage, we have got a function $\bar{h}$ defined on $g(X) \cup\left(\mathbb{R} \backslash B_{f}\right)$ such that the pair $(\bar{f}, \bar{g})$, where $\bar{f}=\bar{h} \circ f$ and $\bar{g}=\bar{h} \circ g$, is an interval order representation of $\prec$, with the additional condition of $\bar{f}:(X, \tau) \rightarrow\left(\mathbb{R}, \tau_{u}\right)$ being continuous.

Now we proceed in an analogous way starting from the pair $(\bar{f}, \bar{g})$. Let $B_{\bar{g}}=\{t \in$ $\bigcup I: I$ is a bad gap of $\bar{g}(X)\}$. Endow $\mathbb{R} \backslash B_{\bar{g}}$ with the topology $\tau_{\leq}$corresponding to the usual ordering $\leq$ on $\mathbb{R} \backslash B_{\bar{g}}$, as a subset of the real line. The inclusion function that goes from $\left(\mathbb{R} \backslash B_{\bar{g}}, \tau_{\leq}\right)$to $\left(\mathbb{R}, \tau_{u}\right)$ is then continuous at every point, excluding those in $g\left(D_{g}\right)$. We may observe that $\mathbb{R} \backslash B_{\bar{g}}$ has neither a left bound nor a right bound. In addition, $\bar{g}(X) \subseteq \mathbb{R} \backslash B_{\bar{g}}$ and both subsets $\bar{g}(X)$ and $\mathbb{R} \backslash B_{\bar{g}}$ have actually the same collection of bad gaps. Moreover the final topology $\tau_{f i n(\bar{g})}$ on $\bar{g}(X)$ corresponding to the function $\bar{g}:(X, \tau) \rightarrow \bar{g}(X)$ is finer than the restriction to $\bar{g}(X)$ of the order topology $\tau_{\leq}$of $\mathbb{R} \backslash B_{\bar{g}}$. Accordingly, any continuous map defined on $\left(\mathbb{R} \backslash B_{\bar{g}}, \tau_{\leq}\right)$will also be be continuous when considered as a map defined on $\left(\bar{g}(X), \tau_{f i n(\bar{g})}\right)$.

Again by the Debreu's Gap Lemma, there exists now a strictly increasing realvalued function $H: \mathbb{R} \backslash B_{\bar{g}} \rightarrow \mathbb{R}$ such that the gaps of $H\left(\mathbb{R} \backslash B_{\bar{g}}\right)$ are all open and, in addition, the function $H:\left(\bar{g}(X), \tau_{f i n(\bar{g})}\right) \rightarrow\left(\mathbb{R}, \tau_{u}\right)$ is continuous. Hence the composition $H \circ \bar{g}:(X, \tau) \rightarrow\left(\mathbb{R}, \tau_{u}\right)$ is also continuous.

At this point, let us see how we can extend $H$ to the bigger domain $\bar{f}(X) \cup(\mathbb{R} \backslash$ $\left.B_{\bar{g}}\right)$ in order to get a new function $\bar{H}$ such that the pair $(\bar{H} \circ \bar{f}, \bar{H} \circ \bar{g})$ is again a representation of $\prec$ as an interval order, with the additional key property that now both $\bar{H} \circ \bar{f}$ and $\bar{H} \circ \bar{g}(=H \circ g)$ are continuous.

\footnotetext{
$\overline{{ }^{2} A \text { denotes here a directed set of indices. }}$
} 
To prove this, fix a point $t \in \bar{f}(X)$. The following situations may occur:

Case 1: If $t \in \bar{f}(X) \bigcap\left(\mathbb{R} \backslash B_{\bar{g}}\right)$, we declare that $\bar{H}(t)=H(t)$. In particular, $\bar{H}$ will become an extension of $H$.

Case 2: If $t \in \bar{f}(X) \bigcap B_{\bar{g}}$ belongs to a bad gap of $\bar{g}(X)$ of the kind $[b, a)$ for some real numbers $a, b$, with $\bar{g}(x)=a$ for some $x \in X$ such that $\bar{g}$ is discontinuous at $x$, then there exists a net $\left(x_{\alpha}\right)_{\alpha \in A}$ in $X$ such that $\bar{g}\left(x_{\alpha}\right)<b<a$ holds for every $\alpha \in A$, and $\left(x_{\alpha}\right)_{\alpha \in A}$ converges to $x$. Since $\prec$ is a suitable semiorder, it follows by part (c) of Lemma 2 that $\{t\}=\bar{f}(X) \bigcap[b, a]$. In this situation, we define $\bar{H}(t)=H(a)$.

Case 3: If $t \in \bar{f}(X) \bigcap B_{\bar{g}}$ belongs to a bad gap of $\bar{g}(X)$ of the kind ( $\left.a, b\right]$ for some real numbers $a, b$, with $\bar{g}(x)=a$ for some $x \in X$ such that $\bar{g}$ is discontinuous at $x$, then $\bar{g}(x)=a<t=\bar{f}(y)$ holds for some $y \in X$. Thus $x \prec y$. Since the semiorder $\prec$ is, by hypothesis, suitable, it is in particular $\tau$-continuous (see part (a) of Lemma 2). Thus, the set $L_{\prec}(y)=\{z \in X: \bar{g}(z)<\bar{f}(y)\}=$ $\bar{g}^{-1}((-\infty, a])=\bar{g}^{-1}([-\infty, \bar{g}(x)))$ is $\tau$-open. Moreover, for any $c \in \mathbb{R}$ such that $c<a=\bar{g}(x)$ it holds that $\bar{g}^{-1}((c, a])=\bar{g}^{-1}((-\infty, \bar{g}(x)]) \cap \bar{g}^{-1}((c,+\infty))$. As a matter of fact, $\bar{g}^{-1}\left((c,+\infty)=\bigcup\left\{U_{\prec^{0}}(z): c<\bar{g}(z)\right\}\right.$ since $\bar{g}$ is a utility function that represents $\precsim^{0}$. Thus $\bar{g}^{-1}((c,+\infty)$ is $\tau$-open because, by hypothesis, the main trace $\precsim^{0}$ had been assumed to be $\tau$-continuous. Therefore $\bar{g}^{-1}((c, a])$ is $\tau$-open for every $c \in \mathbb{R}$ such that $c<a=\bar{g}(x)$. This implies that $\bar{g}$ is continuous at $x$, which contradicts the assumption of $(a, b]$ being a bad gap of $\bar{g}(X)$. In other words, case 3 is impossible.

Once the extension $\bar{H}$ has been defined, we check that $\bar{g}(x)<\bar{f}(y) \Leftrightarrow H(\bar{g}(x))=$ $\bar{H}(\bar{g}(x))<\bar{H}(\bar{f}(y))$ holds true for every $x, y \in X$. To see this, we may observe that if $\bar{f}(y) \in \bar{g}(X)$ then the claim is obvious because $H$ is strictly increasing in $\bar{g}(X)$. Furthermore, if $\bar{f}(y) \notin \bar{g}(X)$ then $\bar{f}(y)$ belongs to a gap $[b, \bar{g}(z)=a$ ) for some $z \in X$ and $a, b \in \mathbb{R}$. Therefore, for any $x \in X$ with $\bar{f}(y)>\bar{g}(x)$ it holds that $\bar{g}(x)<\bar{g}(z)=a$, and then $H(\bar{g}(x))<H(a)=\bar{H}(\bar{f}(y))$.

Notice also that $\bar{H}$ is strictly increasing, not only in $\mathbb{R} \backslash B_{\bar{g}}$ but also in $\bar{f}(X)$, so that both functions $\bar{H} \circ \bar{f}$ and $\bar{H} \circ \bar{g}(=H \circ \bar{g})$ are utility representations for $\precsim^{0}$.

At this stage, we have got a function $\bar{H}$ defined on $\bar{f}(X) \cup\left(\mathbb{R} \backslash B_{\bar{g}}\right)$ such that the pair $(u, v)$, where $u=\bar{H} \circ \bar{f}$ and $v=\bar{H} \circ \bar{g}(=H \circ \bar{g})$, is an interval order representation of $\prec$, with the additional condition of $v$ being continuous.

To finish the proof, let us show that $u$ is also continuous. Notice that $u=\bar{H} \circ \bar{f}$, and $\bar{f}=\bar{h} \circ f(=h \circ f)$ is indeed continuous. Moreover $\bar{H}$ is an extension of $H$, and $H$ is already continuous at any point that lies in $\mathbb{R} \backslash B_{\bar{g}}$. Thus, it remains to check the continuity of $u$ at any point $x \in X$ such that $\bar{f}(x) \in B_{\bar{g}}$. To see this, notice that for some $z \in X$ and $a, b \in \mathbb{R}$, it holds that $\bar{f}(x)$ belongs to a bad gap $[b, a=\bar{g}(z)$ ) of $\bar{g}(X)$. We distinguish two cases: 
Case 1: Either it holds that $\bar{f}(x)=b$, and $b$ is an isolated point of $\bar{f}(X)$ with respect to the restriction to $\bar{f}(X)$ of the usual Euclidean topology on $\mathbb{R}$, or else it holds that $\bar{f}(x) \in(b, a)$. In this case, because of the continuity of $\bar{f}$, we have that the set $\left\{y \in X: y \sim^{0} x\right\}$ is $\tau$-open. Therefore, if a net $\left(x_{\alpha}\right)_{\alpha \in A}$ converges to $x$, there exists an index $\alpha_{0} \in A$ such that for every index $\beta$ with $\alpha_{0} \prec_{A} \beta$, it holds that $x_{\beta} \sim^{0} x$. (Here $\prec_{A}$ stands for the linear order with which the directed set of indices $A$ is endowed). Since $u$ is a utility representation of $\precsim 0$ we have that the net $\left(u\left(x_{\alpha}\right)\right)_{\alpha \in A}$ is quasi-constant. Indeed, for every index $\beta$ with $\alpha_{0} \prec_{A} \beta$, it holds that $u\left(x_{\beta}\right)=u(x)$. Obviously, this implies that the net $\left(u\left(x_{\alpha}\right)\right)_{\alpha \in A}$ converges to $u(x)$, so that $u$ is continuous at $x$.

Case 2: It holds that $\bar{f}(x)=b$, and $b$ is not an isolated point of $\bar{f}(X)$ with respect to the restriction to $\bar{f}(X)$ of the usual Euclidean topology on $\mathbb{R}$, so that for every $\epsilon>0$ we have that $\bar{f}(X) \bigcap(b-\epsilon, b) \neq \emptyset$. Since $\bar{f}$ is already continuous, it is enough to check the continuity of $\bar{H}$ at $b=\bar{f}(x)$. Since we are now working directly on the real line $\mathbb{R}$, there is no loss of generality in considering a strictly increasing sequence $\left(y_{n}\right)_{n \in \mathbb{N}}$ such that $\bar{H}\left(y_{n}\right)$ is defined for every $n \in \mathbb{N}$ and the limit of $y_{n}$ is $b$. Since $\bar{f}(X) \bigcap(b-\epsilon, b) \neq \emptyset$ for every $\epsilon>0$, given $y_{n}$ we may find an element $z_{n} \in \mathbb{R} \backslash B_{\bar{g}}$ such that $y_{n}<z_{n}<b$ Also, given $z_{n}$, there exists $k \in \mathbb{N}$ such that $z_{n}<y_{n+k}<b$. Thus, the sequence $\left(z_{n}\right)_{n \in \mathbb{N}}$ also converges to $b$. Notice now that $H$ is continuous and strictly increasing in $\mathbb{R} \backslash B_{\bar{g}}$, and for any strictly increasing sequence $\left(z_{n}\right)_{n \in \mathbb{N}}$ in $\mathbb{R} \backslash B_{\bar{g}}$ that converges to $b$ it holds that $\left(H\left(z_{n}\right)\right)_{n \in \mathbb{N}}$ converges to $H(a)=\bar{H}(b)=\bar{H}(\bar{f}(x))$. Hence the sequence $\bar{H}\left(y_{n}\right)_{n \in \mathbb{N}}$ also converges to $H(a)=\bar{H}(b)=\bar{H}(\bar{f}(x))$, so that $\bar{H}$ is indeed continuous at $b$.

This concludes the proof.

The hypotheses of the statement of Theorem 5 do not force the topology $\tau$ to be natural, as next Example 4 shows. Therefore, Theorem 5 has a different scope that the main results issued for natural topologies (see Theorem 3 (b) above). ${ }^{1}$

Example 4. Let $X=(-1.5,-0.8) \cup(-0.5,0.2) \cup[0.5,0.85] \cup\{1.3\}$ endowed with the semiorder $\prec$ given by $x \prec y \Leftrightarrow x+1<y$, for every $x, y \in X$. It can be seen that $\prec^{0}$ coincides here with the usual Euclidean order $(<)$. Let us consider on $X$ the order topology $\tau_{\precsim 0}$ induced by $\precsim^{0}$. That topology $\tau_{\precsim 0}$ is compatible as regards the $\sim^{0}$. Moreover, the semiorder $\prec$ is suitable as regards $\tau_{\precsim 0}$. Furthermore, the strict upper contour $U_{\prec^{*}}(0)$ is $[0.5,0.85] \cup\{1.3\}$, which fails to be $\tau_{\precsim 0}$-open, so that, considering $\prec$ as an interval order, the topology $\tau_{\precsim 0}$ is not natural. In addition, in this case there exists a continuous Scott-Suppes representation $(u, 1)$ of the semiorder $\prec$ such that $u$ also represents the main trace $\precsim^{0}$. Indeed, to that extent we may use the function $u: X \rightarrow \mathbb{R}$ given by $u(x)=\frac{10 x}{7}+\frac{9}{14}$ if $x<-0.8 ; u(x)=\frac{10 x}{7}+\frac{3}{14}$ if $-0.5<x<0.2$; $u(x)=\frac{10 x}{7}-\frac{3}{14}$ if $0.5 \leq x \leq 0.85$ and $u(1.3)=1.5$. 


\section{Remark 10.}

(1) By adding some more hypotheses to the statement of Theorem 5 we could finally prove that the semiorder $\prec$ on the topological space $(X, \tau)$ actually has a continuous Scott-Suppes representation. If $(u, v)$ is a pair of continuous functions that represents the semiorder $\prec$ as an interval order, a possible idea is searching for a continuous increasing function $h: \mathbb{R} \rightarrow \mathbb{R}$ such that $h(v(x))=$ $h(u(x))+1$ for every $x \in X$, so that the pair $(h \circ u, 1)$ constitutes a continuous Scott-Suppes representation of $\prec$. Indeed, it has already been proved that this idea works, at least, when $(X, \tau)$ is connected, the traces $\prec^{*}$ and $\prec^{* *}$ agree, and there is no singular element ${ }^{3}$ as regards $\prec .{ }^{34,46}$

(2) With the hypotheses that appear in the statement of Theorem 5, the result achieved cannot be improved. This can be shown by means of Example 5 in Ref. 32, namely, let $X=(-\infty,-1) \cup(-0.5,0) \cup[0.5,1) \cup(1.5,+\infty) \subset \mathbb{R}$. Endow $X$ with the semiorder $\prec$ given by $x \prec y \Leftrightarrow x+1<y(x, y \in X)$. Observe that $\prec$ is representable in the sense of Scott-Suppes, by means of the pair $(\iota, 1)$, where $\iota: X \rightarrow \mathbb{R}$ is the inclusion. Consider on $X$ the topology $\tau$ defined by means of the subbasis $\{\emptyset\} \cup\{X\} \cup\{z \in X: z<x\}_{x \in X} \cup\{y \in X: x<y\}_{x \in X}$, where $<$ stands for the usual strict order of $\mathbb{R}$. It has been proved that the semiorder $\prec$ does not admit a continuous Scott-Suppes representation if we endow $X$ with the topology $\tau$ and the real line $\mathbb{R}$ with the usual topology. In addition, $\prec$ is suitable as regards $\tau$, and the topology $\tau$ is compatible with respect to the indifference of the main trace of $\prec$ because, as an striaghtforward checking shows, the main trace $\prec^{0}$ coincides here with the usual strict order $<$ of the real line $\mathbb{R}^{32}$

\section{Continuous representability of interval orders: the finite case}

Some characterizations of the continuous Scott-Suppes representability of semiorders on a topological space $(X, \tau)$ in which the set $X$ is finite were already obtained. ${ }^{32}$

In this section we achieve some results in a parallel direction. Namely, we characterize the representability, through a pair $(u, v)$ of continuous real-valued functions, of interval orders defined on a topological space $(X, \tau)$ with $X$ finite.

\subsection{Necessary conditions for the continuous representability of interval orders}

Lemma 2 above was introduced in Ref. 32 to deal with the continuous Scott-Suppes representability of semiorders. In an analogous way, we furnish now several necessary conditions for the representability of an interval order through a pair of continuous

\footnotetext{
${ }^{3}$ An element $x \in X$ is called singular with respect to a semiorder $\prec$ if for every $y, z \in X$ it holds that $[(x \sim y) \wedge(x \sim z)] \Rightarrow(y \sim z)$.
} 
real-valued functions, in the general case. To do so, we start with the following preparatory lemma.

Lemma 4. Let $\prec$ denote an interval order defined on a nonempty set $X$. Assume that $\prec$ is representable by means of a pair $(u, v)$ of real-valued functions. Then, the following statements hold true for any $x, y \in X$ :

(a) $x \prec^{*} y \Longrightarrow v(x)<v(y)$,

(b) $x \prec^{* *} y \Longrightarrow u(x)<u(y)$,

(c) $v(x)=v(y) \Longrightarrow x \sim^{*} y$,

(d) $u(x)=u(y) \Longrightarrow x \sim^{* *} y$.

Proof. Indeed, for any $x, y \in X$ with $x \prec^{*} y$ it follows, by definition, that there exists $z \in X$ such that $x \prec z \precsim y$. Hence we have that $v(x)<u(z) \leq v(y)$. Therefore, $v(x)<v(y)$.

In the same way, for any $x, y \in X$ with $x \prec^{* *} y$ it follows, by definition, that there exists $z \in X$ such that $x \precsim z \prec y$. Hence $u(x) \leq v(z)<u(y)$, and consequently $u(x)<u(y)$.

Finally, part $c$ (respectively, $d$ ) follows from part $a$ (respectively, from $b$ ).

We are now ready to introduce some necessary conditions for the continuous representability of an interval order (in the general case).

Proposition 2. Let $(X, \tau)$ a topological space endowed with an interval order $\prec$. If the order interval is representable through a pair $(u, v)$ of continuous real-valued functions, then the following properties hold true:

(a) The interval order is $\tau$-continuous.

(b) If a net $\left(x_{j}\right)_{j \in J} \subseteq X$ converges to two points $a, b \in X$, then $a \sim^{0} b$.

(c) If a net $\left(x_{j}\right)_{j \in J} \subseteq X$ converges to $a \in X$, and $b, c \in X$ are such that $x_{j} \prec b \precsim a$ and $x_{j} \prec c \precsim a$ for any $j \in J$, then $b \sim^{* *} c$.

(d) If a net $\left(x_{j}\right)_{j \in J} \subseteq X$ converges to $a \in X$, and $b, c \in X$ are such that $a \precsim b \prec x_{j}$ and $a \precsim c \prec x_{j}$ for any $j \in J$, then $b \sim^{*} c$.

Proof. Assume that $\prec$ is representable through a pair $(u, v)$ of continuous maps. (a) (We prove this property by using the characterization of $\tau$-continuity by means of nets, see e.g. Lemma 2 in Ref. 32).

Let $\left(x_{j}\right)_{j \in J}$ be a net that converges to $a \in X$. Suppose that there exits $b \in X$ such that $x_{j} \precsim b$ for any $j \in J$. It follows now that $u\left(x_{j}\right) \leq v(b)$ for any $j \in J$. Thus, $u(a)=\lim _{j \in J} u\left(x_{j}\right) \leq v(b)$, which implies that $a \precsim b$. (A similar argument is used to handle the case in which $b \precsim x_{j}$ for any $\left.j \in J\right)$.

Consequently, the interval order is $\tau$-continuous.

(b) Let $\left(x_{j}\right)_{j \in J} \subseteq X$ be a net that converges to two points $a, b \in X$. Then, since $u$ is continuous, the image of the net $\left(u\left(x_{j}\right)\right)_{j \in J}$ converges to $u(a)$ and also to $u(b)$ as regards the usual topology of $\mathbb{R}$. By the same reason, the net $\left(v\left(x_{j}\right)\right)_{j \in J}$ converges to 
$v(a)$ and to $v(b)$. Endowed with the usual topology the real line is a Hausdorff space, so that a convergent net has a unique limit. Hence $u(a)=u(b)$ and $v(a)=v(b)$. Therefore, by Lemma 4, we conclude that $a \sim^{*} b$ and $a \sim^{* *} b$. This implies that $a \sim^{0} b$.

(c) Let $\left(x_{j}\right)_{j \in J} \subseteq X$ be a net that converges to $a \in X$. Let $b, c \in X$ be such that $x_{j} \prec b \precsim a$ and $x_{j} \prec c \precsim a$ hold for any $j \in J$. Then we have that $v\left(x_{j}\right)<u(b) \leq$ $v(a)$ and $v\left(x_{j}\right)<u(c) \leq v(a)$ hold for any $j \in J$. By continuity of $u$ and $v$, it follows that $v(a) \leq u(b) \leq v(a)$ and $v(a) \leq u(c) \leq v(a)$. Therefore, $v(a)=u(b)=u(c)$. By Lemma 4 again, we conclude that $b \sim^{* *} c$.

(d) This can be proved in a similar way to $(c)$.

\subsection{Characterization of the continuous representability of interval orders on a finite topological space}

Let us prove now that when $(X, \tau)$ is a topological space with $X$ finite, the necessary conditions introduced in Proposition 2 are, actually, sufficient.

Theorem 6. Let $(X, \tau)$ be a topological space in which the set $X$ is finite. Let $\prec$ be an interval order defined on $X$. The following statements hold true:

(a) The interval order $\prec$ has a representation by means of a pair $(u, v)$ of continuous real-valued functions.

(b) The interval order $\prec$ satisfies the necessary conditions (a)-(d) introduced in Proposition 2.

(c) The interval order $\prec$ satisfies the condition (b) introduced in Proposition 2, namely, if a net $\left(x_{j}\right)_{j \in J} \subseteq X$ converges to two points $a, b \in X$, then $a \sim^{0} b$.

(d) The topology $\tau$ is natural with respect to the interval order $\prec$.

Proof. We will follow this scheme: $(\mathrm{a}) \Longrightarrow(\mathrm{b}) \Longrightarrow(\mathrm{c}) \Longrightarrow(\mathrm{a}) ;(\mathrm{d}) \Longrightarrow$ (a) and (c) $\Longrightarrow(\mathrm{d})$.

The implication $(\mathrm{a}) \Longrightarrow(\mathrm{b})$ has already been stated for the general case, in Proposition 2. The implication (b) $\Longrightarrow$ (c) is trivial.

To prove that $(\mathrm{c}) \Longrightarrow(\mathrm{a})$, we consider a representation of $\prec$ through a pair $(u, v)$ such that, in addition, $u$ represents the total preorder ${ }^{* *}$ whereas $v$ represents $\precsim *$. (Such a pair $(u, v)$ exists by Theorem 3 in Ref. 39).

Let us see now that the functions $u$ and $v$ are indeed continuous with respect the given topology $\tau$ on $X$ and the usual topology on the real line $\mathbb{R}$. To do so, consider a net $\left(x_{j}\right)_{j \in J}$ that converges to a point $a \in X$. Since the set $X$ is, by hypothesis, finite, there exists a constant subnet $\left(c=x_{k}\right)_{k \in K ; K \subseteq J}$, that also converges to $a$ because it is a subnet of the net $\left(x_{j}\right)_{j \in J}$ which, by assumption, converges to $a \in X$.

Since the constant subnet $(c)_{k \in K}$ also converges to $c$, by the hypothesis (c) it follows that $a \sim^{*} c$ and $a \sim^{* *} c$.

Furthermore, there exists $j_{0} \in J$ such that, for every index $i \in J$ with $j_{0} \prec_{J} i$ (where $\prec_{J}$ stands for the order on the directed set $J$ ) there exists a constant subnet, 
of the given net $\left(x_{j}\right)_{j \in J}$, whose constant term is $x_{i}$. Again, this is a consequence of $X$ being finite. Therefore, by (c) there exists an index $j_{0} \in J$ such that $x_{i} \sim^{*} a$ and $a \sim^{* *} x_{i}$ hold for every $j_{0} \prec_{J} i$.

Since $u$ represents $\precsim^{* *}$ and $v$ represents $\precsim^{*}$ it follows that $u(a)=u(c)=u\left(x_{i}\right)$, and also $v(a)=v(c)=v\left(x_{i}\right)$, for every $j_{0} \prec_{J} i$. Consequently, the nets $\left(u\left(x_{j}\right)\right)_{j \in J}$ and $\left(v\left(x_{j}\right)\right)_{j \in J}$ are respectively convergent to $u(a)$ and $v(a)$ as regards the usual topology on the real line $\mathbb{R}$. Thus we conclude that both the functions $u$ and $v$ are continuous with respect to the given topology $\tau$ on $X$ and the usual topology on $\mathbb{R}$.

The fact $(\mathrm{d}) \Longrightarrow(\mathrm{a})$ follows from Theorem 3 , using that $X$ is finite.

To conclude, let us prove now that $(\mathrm{c}) \Longrightarrow(\mathrm{d})$. By the equivalence $(\mathrm{c}) \Longleftrightarrow(\mathrm{b})$, we already now that $\prec$ is $\tau$-continuous. Let us prove now that the traves $\prec *$ and $\prec^{* *}$ are also $\tau$-continuous. To do so, assume by contradiction that $\precsim^{*}$ fails to be $\tau$ continuous, so that there exists $x \in X$ such that $L_{\prec *}(x)=\left\{z \in X: z \prec^{*} x\right\}$, or else $U_{\prec^{*}}(x)=\left\{y \in X: x \prec^{*} y\right\}$ is not $\tau$-open. Assuming that $L_{\prec^{*}}(x)$ is not open, there exists a net $\left(x_{j}\right)_{j \in J} \subseteq\left\{t \in X: x \precsim^{*} t\right\}$ that converges to an element $a \in L_{\prec^{*}}(x)$. That is, we have that $a \prec^{*} x \precsim^{*} x_{j}$ for every $j \in J$, and, in addition, the net $\left(x_{j}\right)_{j \in J}$ converges to $a$. Since $X$ is finite, there is a constant subnet $(c)_{k \in K ; K \subseteq J}$ that, because it is a subnet of $\left(x_{j}\right)_{j \in J}$, it converges to $a$, too. And, obviously, this constant subnet converges to $c$. Thus we get $a \prec^{*} x \precsim^{*} c$ and $(c)_{k \in K}$ converges both to $a$ and $c$. But, since $a \prec^{*} c$, this contradicts the hypothesis $(c)$. The case in which $U_{\prec *}(x)$ fails to be $\tau$-open is handled in an entirely analogous way. Finally, the $\tau$-continuity of the other trace, namely $\prec^{* *}$ is also proved by means of similar arguments.

\section{Concluding remarks}

Alternatively to the techniques based on the concept of a natural topology with respect to an interval order, introduced in the seminal reference Ref. 1, new results on the representability of interval orders through a pair of continuous real-valued functions have been obtained. A key to this achievement is the concept of a topology $\tau$ compatible with the indifference $\sim^{0}$ of the main trace of an interval order $\prec$ defined on a given nonempty set $X$. In the particular case in which $X$ is finite, a full characterization of the continuous representability of interval orders has been obtained. The independence (in the general case) of the techniques introduced here and the ones that lean on the notion of a natural topology, launched in Ref. 1, has also been shown (see Example 4 above). However, when $X$ is finite both techniques could indeed be considered as equivalent, as Theorem 6 shows.

The characterization of the continuous representability of interval orders and semiorders still remain open for the general case, but, at least, we have already got characterizations (in Ref. 32 for semiorders and in Theorem 6 above for interval orders) for the case in which the supporting set is finite. 


\section{Acknowledgements}

The authors acknowledge financial support from the Ministry of Economy and Competitiveness of Spain under grant MTM2012-37894-C02-02, and from the University of the Basque Country UPV/EHU under grant UFI11/52.

\section{References}

1. G. Bosi, M.J. Campión, J.C. Candeal and E. Induráin, Interval-valued representability of qualitative data: the continuous case, Internat. J. Uncertain. Fuzziness KnowledgeBased Systems 15 (3) (2007) 299-319.

2. D.S. Bridges and G.B. Mehta, Representations of Preference Orderings, SpringerVerlag, Berlin-Heidelberg-New York, 1995.

3. G. Cantor, Beiträge zur Begründung der transfiniten Mengenlehre I, Math. Ann. 46 (1895) 481-512.

4. G. Cantor, Beiträge zur Begründung der transfiniten Mengenlehre II, Math. Ann. 49 (1895) 207-246.

5. G. Birkhoff, Lattice Theory (third edition), American Mathematical Society, Providence, RI, 1967.

6. A. Tversky, Intransitivity of preferences, Psychological Rev. 76(1) (1969) 31-48.

7. P.C. Fishburn, Intransitive indifference with unequal indifference intervals, J. Math. Psych. 7 (1970) 144-149.

8. P.C. Fishburn, Intransitive indifference in preference theory: a survey, Oper. Res. 18(2) (1970) 207-228.

9. D.S. Bridges, Numerical representations of intransitive preferences on a countable set, J. Econom. Theory 30 (1983) 213-217.

10. G. Bosi and R. Isler, Representing preferences with nontransitive indiffference by a single real-valued function, J. Math. Econom. 24 (1995) 621-631.

11. R.D. Luce, Semiorders and a theory of utility discrimination, Econometrica 24 (1956) 178-191.

12. D. Scott and P. Suppes, Foundational aspects of theories of measurement, J. Symbolic Logic 23 (1958) 113-128.

13. J.C. Candeal and E. Induráin, Semiorders and thresholds of utility discrimination: solving the Scott-Suppes representability problem, J. Math. Psych. 54 (2010) 485490 .

14. N. Wiener, Contribution to the theory of relative position, Math. Proc. Cambridge Philos. Soc. 17 (1914) 441-449.

15. N. Wiener, A new theory of measurement, Proc. London Math. Soc. 19 (1919-1920) 181-205.

16. P.C. Fishburn and B. Monjardet, Norbert Wiener on the theory of measurement (1914, 1915, 1921), J. Math. Psychol. 36 (1992) 165-184.

17. P.C. Fishburn, Utility Theory for Decision-Making, Wiley, New York, 1970.

18. P.C. Fishburn, Interval representations for interval orders and semiorders, J. Math. Psych. 10 (1973) 91-105.

19. P.C. Fishburn, Interval Orders and Interval Graphs, Wiley, New York, 1985.

20. M.J. Campión, J.C. Candeal and E. Induráin, On Yi's extension property for totally preordered topological spaces, J. Korean Math. Soc. 43 (1) (2006), 159-181.

21. M.J. Campión, J.C. Candeal and E. Induráin, Semicontinuous planar total preorders on non-separable metric spaces, J. Korean Math. Soc. 46 (4) (2009), 701-711. 
22. M.J. Campión, J.C. Candeal, E. Induráin and G.B. Mehta, Continuous order representability properties of topological spaces and algebraic structures, J. Korean Math. Soc. 49 (3) (2012), 449-473.

23. J.C. Candeal, E. Induráin and M. Sanchis, Order representability in groups and vector spaces, Expo. Math. 30 (2012) 103-123.

24. G. Bosi and M.E. Zuanon, Upper semicontinuous representations of interval orders, Math. Social Sci. 68 (2014) 60-63.

25. D.S. Bridges, Numerical representation of interval orders on a topological space, $J$. Econom. Theory 38 (1986) 160-166.

26. A. Chateauneuf, Continuous representation of a preference relation on a connected topological space, J. Math. Econom. 16 (1987) 139-146.

27. G. Bosi, Continuous representations of interval orders based on induced preorders, Rivista di Matematica per le Scienze Economiche e Soziali 18 (1) (1995) 75-82.

28. J.C. Candeal, E. Induráin and M. Zudaire, Continuous representability of interval orders, Appl. Gen. Topol. 5 (2) (2004) 213-230.

29. G. Bosi, J.C. Candeal and E. Induráin, Continuous representability of interval orders and biorders, J. Math. Psych. 51 (2007) 122-125.

30. G. Bosi and M.E. Zuanon, Semicontinuous representability of interval orders on a metrizable topological space, Int. J. Contemp. Math. Sciences 2 (18) (2007) 853858.

31. G. Bosi and M.E. Zuanon, Representations of an interval order by means of two upper semicontinuous functions, Int. Math. Forum 6 (42) (2011) 2067-2071.

32. A. Estevan, J. Gutiérrez García and E. Induráin, Further results on the continuous representability of semiorders, Internat. J. Uncertain. Fuzziness Knowledge-Based Systems 21 (5) (2013). 675-694.

33. S.H. Gensemer, Continuous semiorder representations, J. Math. Econom. 16 (1987) 275-289.

34. M.J. Campión, J.C. Candeal, E. Induráin and M. Zudaire, Continuous representability of semiorders, J. Math. Psych. 52 (2008) 48-54.

35. E. Olóriz, J.C. Candeal and E. Induráin, Representability of interval orders, $J$. Econom. Theory 78 (1) (1998) 219-227.

36. J.C. Candeal, E. Induráin and M. Zudaire, Numerical representability of semiorders, Math. Social Sci. 43 (1) (2002) 61-77.

37. F. Aleskerov, D. Bouyssou and B. Monjardet, Utility maximization, choice and preference (second edition), Springer, Berlin, 2007.

38. B. Monjardet, Axiomatiques et propriétés des quasi-ordres, Math. Sci. Hum. 63 (1978) $51-82$.

39. G. Bosi, J.C. Candeal, E. Induráin, E. Olóriz and M. Zudaire, Numerical representations of interval orders, Order 18 (2001) 171-190.

40. J.C. Candeal, A. Estevan, J. Gutiérrez-García and E. Induráin, Semiorders with separability properties, J. Math. Psychol. 56 (2012) 444-451.

41. K.L. Manders, On JND representations of semiorders, J. Math. Psychol. 24 (1981) 224-248.

42. A. Beja and I. Gilboa, Numerical representations of imperfectly ordered preferences. A unified geometric exposition, J. Math. Psych. 36 (1992) 426-449.

43. G. Debreu, Representation of a preference ordering by a numerical function, In R. Thrall, C. Coombs and R. Davies (Eds.), Decision processes, John Wiley, New York, 1954.

44. G. Debreu, Continuity Properties of Paretian Utility, Internat. Econom. Rev. 5 (1964) 285-293. 
April 23, 2014 13:22 WSPC/INSTRUCTION FILE "IJUFKS Bosi et al. (APRIL 2014)"

45. P. Ouwehand, A simple proof of Debreu' s gap lemma, Orion 26 (1) (2010) 17-20.

46. F.J. Abrísqueta, J.C. Candeal, R.G. Catalán, J.R. De Miguel and E. Induráin, Generalized Abel functional equations and numerical representability of semiorders, Publ. Math. Debrecen 78 (3-4) (2011) 557-568. 